\title{
Interferometers for Displacement-Noise-Free Gravitational-Wave Detection
}

\author{
Yanbei Chen, Archana Pai, and Kentaro Somiya \\ Max-Planck Institut für Gravitationsphysik, Am Mühlenberg 1, 14476 Potsdam, Germany \\ Seiji Kawamura and Shuichi Sato \\ TAMA project, National Astronomical Observatory of Japan, 2-21-1 Osawa, Mitaka, Tokyo 181-8588, Japan \\ Keiko Kokeyama \\ Ochanomizu University, 2-1-1, Otsuka, Bunkyo-ku, Tokyo, 112-8610 Japan
}

Robert L. Ward

LIGO Project 18-34, California Institute of Technology, Pasadena, California 91125, USA

Keisuke Goda and Eugeniy E. Mikhailov

LIGO Laboratory, Massachusetts Institute of Technology, Cambridge, Massachusetts 02139, USA

(Received 25 May 2006; published 13 October 2006)

\begin{abstract}
We propose a class of displacement- and laser-noise-free gravitational-wave-interferometer configurations, which does not sense nongeodesic mirror motion and laser noise, but provides a nonvanishing gravitational-wave signal. Our interferometers consist of four mirrors and two beam splitters, which form four Mach-Zehnder interferometers. By contrast to previous works, no composite mirrors with multiple reflective surfaces are required. Each mirror in our configuration is sensed redundantly, by at least two pairs of incident and reflected beams. Displacement- and laser-noise-free detection is achieved when output signals from these four interferometers are combined appropriately. Our 3-dimensional interferometer configuration has a low-frequency response proportional to $f^{2}$, which is better than the $f^{3}$ achievable by previous 2-dimensional configurations.
\end{abstract}

It was recently demonstrated theoretically that gravitational-wave $(\mathrm{GW})$ detection does not require freely falling test masses, because nongeodesic test-mass motion affects the travel times of pulses only when they arrive and leave the test masses, while the effect of GWs is distributed [1]. This idea was further explored in Ref. [2], which shows that once the number $\mathcal{N}$ of test masses is large enough, the total number of available light-pulse-travel-time measurement channels $\left[O\left(\mathcal{N}^{2}\right)\right]$ will exceed that of clock- and displacement-noise channels $[O(\mathcal{N})]$, and there must exist clock- and displacement-noise-free channels. Reference [2] also showed that interferometers can be combined to realize displacement- and laser-noise-free GW detection. As argued there, when lasers are used as clocks, motions of laser devices cause a Doppler shift to laser frequencies indistinguishable from laser noises. Therefore, displacement-noise-free detection, strictly speaking, requires the cancellation of laser noise. Henceforth, we shall use the term displacement-noise-free interferometry (DFI) to describe displacement- and laser-noise-free interferometer configurations.

References [1,2] study DFI by calculating pulse time delays between emitters and receivers fixed on point test masses. This approach, although mathematically simpler and in principle applicable to laser interferometry, does not provide practical interferometer configurations right away. Configurations constructed so far require composite mir- rors, namely, mirrors with multiple reflective surfaces. Apart from being experimentally challenging, the use of composite mirrors gives rise to the fundamental difficulty that thermal fluctuations of relative positions between the multiple reflective surfaces are not canceled. In addition, so far only 2-dimensional configurations have been explored, for which it can be proved that shot-noise-limited sensitivity to GWs can be no better than $\sim f^{3}$ in low frequencies [3].

In this Letter, we propose simple 2D and 3D configurations that implement DFI without composite mirrors. We use Mach-Zehnder interferometers (MZs) as building blocks, with laser noises canceled right in the beginning. Moreover, each mirror participates in at least two MZs, and thus has its location sensed redundantly. Finally, by combining the MZ output signals, we are able to cancel amongst the redundant displacement information, leaving nonvanishing response to GWs. In particular, we will show that our 3D configuration has $\sim f^{2}$ sensitivity in low frequencies, which is the best one can achieve with DFI [3]. The 2D configuration, which has $\sim f^{3}$ response in low frequencies, is proposed mainly for the purpose of initial experimental tests.

$G W$ response of a plane electromagnetic (EM) wave.Here we provide a brief derivation of the GW-induced phase shift of light. A weak plane GW on Minkowski background can be described with a metric 


$$
g_{\mu \nu}=\eta_{\mu \nu}+h_{\mu \nu}
$$

in the Cartesian coordinate system, $x^{\mu}=(c t, \mathbf{x})$, with $t$ the time coordinate, $\mathbf{x}$ the spatial coordinates, $c$ the speed of light, and $\eta_{\mu \nu}=\operatorname{diag}(-1,1,1,1)$. In the transversetraceless (TT) gauge, $h_{\mu \nu}$ only has spatial components:

$$
\begin{aligned}
\mathbf{h}^{\mathrm{TT}}(t, \mathbf{x})= & h_{+}\left(t-\mathbf{e}_{Z} \cdot \mathbf{x} / c\right)\left[\mathbf{e}_{X} \otimes \mathbf{e}_{X}-\mathbf{e}_{Y} \otimes \mathbf{e}_{Y}\right] \\
& +h_{\times}\left(t-\mathbf{e}_{Z} \cdot \mathbf{x} / c\right)\left[\mathbf{e}_{X} \otimes \mathbf{e}_{Y}+\mathbf{e}_{Y} \otimes \mathbf{e}_{X}\right] .
\end{aligned}
$$

Here $\left(\mathbf{e}_{X}, \mathbf{e}_{Y}, \mathbf{e}_{Z}\right)$ is a spatial orthonormal set, with $\mathbf{e}_{Z}$ the wave propagation direction (Fig. 2). We approximate the electromagnetic (EM) wave as a scalar wave, with amplitude

$$
\Phi\left(x^{\mu}\right)=\Phi^{(0)}\left(x^{\mu}\right)\left[1+i \phi^{\mathrm{gw}}\left(x^{\mu}\right)\right],
$$

where $\Phi^{(0)}\left(x^{\mu}\right)=A \exp \left(i k_{\mu} x^{\mu}\right)$ is the 0 th order EM wave when there is no GW ( $A$ is constant), and $\phi^{\mathrm{gw}}\left(x^{\mu}\right)$ the additional phase shift caused by the GW. The EM wave equation $g^{\mu \nu} \Phi_{; \mu \nu}=0$, expanded to leading order in $h_{\mu \nu}$ and $\phi^{\mathrm{gw}}$, can be written as $\eta^{\mu \nu} \Phi_{, \mu \nu}=-h^{\mu \nu} \Phi_{\text {, }}^{(0)}$, where the Lorenz gauge condition $h_{, \nu}^{\mu \nu}=0$ has been used. Because $\phi^{\mathrm{gw}}$ is slowly varying compared to $\Phi^{(0)}$, we can ignore terms like $\phi_{, \mu \nu}^{\mathrm{gw}}$ on the left-hand side, and obtain

$$
k^{\nu} \phi_{, \nu}^{\mathrm{gw}}=h_{\mu \nu} k^{\mu} k^{\nu} / 2,
$$

which accumulates along the null ray in Minkowski spacetime. In particular, if the Minkowski ray starts from $\left(c t_{0}\right.$, $\left.\mathbf{x}_{0}\right)$ and ends at $(c t, \mathbf{x})$, with $\left|\mathbf{x}-\mathbf{x}_{0}\right|=c\left|t-t_{0}\right|=l$ and $\mathbf{N} \equiv\left(\mathbf{x}-\mathbf{x}_{0}\right) / l$, then the GW-induced phase shift is

$$
\phi^{\mathrm{gw}}=\omega l /(2 c) \int_{0}^{1} d \zeta h_{i j}^{\mathrm{TT}}\left(t_{0}+l \zeta, \mathbf{x}_{0}+\mathbf{N} l \zeta\right) N_{i} N_{j} .
$$

$3 D$ Configuration. - We now discuss our 3D configuration (Fig. 1), with mirrors located on the 8 vertices of a regular octahedron, and light beams propagating along its edges, each with length $2 L$. We attach a Cartesian coordinate system to the octahedron, with origin coinciding with its center, $z$ axis coinciding with its $B-A$ axis, $x$ axis parallel to the $C_{1}-D_{1}\left(D_{2}-C_{2}\right)$ direction, and $y$ axis parallel to the $C_{2}-D_{1}\left(D_{2}-C_{1}\right)$ direction. (We have also defined $\xi$ and $\eta$ directions, as shown in the figure.) A 50-50 beam splitter each is located on the vertices $A$ and $B$, with normal directions parallel to the $x$ axis. The four perfectly reflective mirrors at $C_{1,2}$ and $D_{1,2}$ are oriented such that light beams from $A$ are reflected directly to $B$. We assume all perfect mirrors to have amplitude reflectivity $r=1$, and both beam splitters to have $-1 / \sqrt{2}$ amplitude reflectivity for light incident from the $+x$ side (i.e., traveling toward $-x$ direction), and $+1 / \sqrt{2}$ amplitude reflectivity for light incident from the $-x$ side; the edge length is assumed to be an integer multiple of the optical wavelength, at the zero point of the device (i.e., in absence of laser noise, mirror motion, and GW).

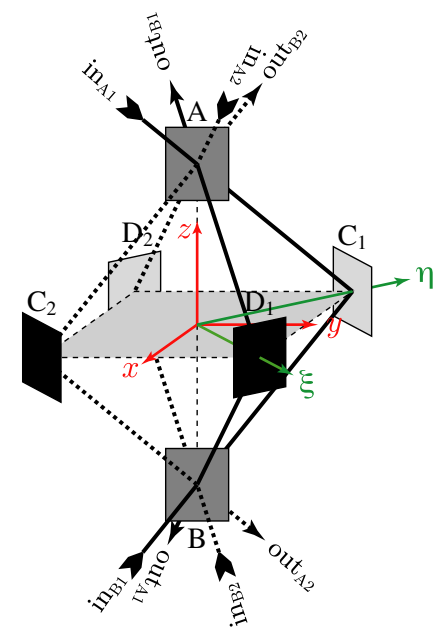

FIG. 1 (color online). The 3D octahedron configuration, which consists of four $\mathrm{MZs} ; A_{1}: A_{t} C_{1} B_{r}-A_{r} D_{1} B_{t}, B_{1}: B_{t} C_{1} A_{r}-$ $B_{r} D_{1} A_{t}$ (solid lines) and $A_{2}: A_{t} D_{2} B_{r}-A_{r} C_{2} B_{t}, B_{2}: B_{t} D_{2} A_{r}-$ $B_{r} C_{2} A_{t}$ (dashed lines). The subscripts $r, t$ indicate reflection and transmission, respectively.

With this setup, we construct four MZ interferometers, $A_{1}, B_{1}$ (light paths in solid lines), $A_{2}$ and $B_{2}$ (light paths in dashed lines), with input and output ports indicated in Fig. 1. At the zero point, the output ports $\left(\right.$ out $_{A_{1}, A_{2}, B_{1}, B_{2}}$ ) are kept dark, while each input port is also the bright port for another interferometer. During operations, for each $\mathrm{MZ}, I=A_{1}, A_{2}, B_{1}, B_{2}$, if $\phi_{I}^{(t)}$ and $\phi_{I}^{(r)}$ represent the additional phase shifts gained by the beams transmitted and reflected from its first beam splitter, respectively, then the output optical amplitude is proportional to

$$
e^{i \phi_{I}^{(t)}}-e^{i \phi_{I}^{(r)}} \propto \phi_{I}^{(t)}-\phi_{I}^{(r)} \equiv \delta \phi_{I} .
$$

For interferometers $A_{1}$ and $A_{2}$, the "first beam splitter" means $A$, while for $B_{1}$ and $B_{2}$, it means $B$. In Eq. (6), we have a minus sign in front of $e^{i \phi_{I}^{(r)}}$, because light always incident from the $+x$ side of the beam splitters, with the first reflection always encounters a $-1 / \sqrt{2}$ reflectivity.

These additional phase shifts can arise from laser noise, displacement noise, and GWs. Because we only consider linear order in GWs and the noises, we can first include only effects of laser and displacement noises, construct a

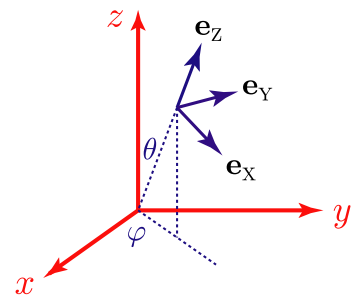

FIG. 2 (color online). The orthonormal system $\left(\mathbf{e}_{X}, \mathbf{e}_{Y}, \mathbf{e}_{Z}\right)$ used to describe a generic plane $\mathrm{GW} ; \mathbf{e}_{Z}$ is the propagation direction. 
combination from the outputs of the four MZs free from these noises, and then calculate its response to GWs. For dark-port detection, each $\mathrm{MZ}$ is already free from laser noise; we only need to consider displacement noise. For a mirror with normal direction $\mathbf{n}$ and incident wave vector $\mathbf{k}$, the phase shift gained by the reflected light when the mirror moves spatially by $\delta \mathbf{x}$ is $2(\mathbf{n} \cdot \mathbf{k})(\mathbf{n} \cdot \delta \mathbf{x})$. For $A_{1}$ and $B_{1}$ interferometers, we have

$$
\begin{gathered}
\phi_{A_{1}}^{(t)}(t)=\sqrt{2} \omega / c\left[\eta_{C_{1}}(t-2 L / c)+x_{B}(t)\right], \\
\phi_{A_{1}}^{(r)}(t)=\sqrt{2} \omega / c\left[\xi_{D_{1}}(t-2 L / c)-x_{A}(t-4 L / c)\right], \\
\phi_{B_{1}}^{(t)}(t)=\sqrt{2} \omega / c\left[\eta_{C_{1}}(t-2 L / c)+x_{A}(t)\right], \\
\phi_{B_{1}}^{(r)}(t)=\sqrt{2} \omega / c\left[\xi_{D_{1}}(t-2 L / c)-x_{B}(t-4 L / c)\right] .
\end{gathered}
$$

Here, $\eta_{C_{1}}$ denotes the motion of $C_{1}$ along the $\eta$ axis and so on. Thus we have

$$
\begin{aligned}
\delta \phi_{A_{1}}-\delta \phi_{B_{1}}= & {\left[\phi_{A_{1}}^{(t)}-\phi_{A_{1}}^{(r)}\right]-\left[\phi_{B_{1}}^{(t)}-\phi_{B_{1}}^{(r)}\right] } \\
= & \sqrt{2} \omega / c\left[x_{B}(t)-x_{A}(t)-x_{B}(t-4 L / c)\right. \\
& \left.+x_{A}(t-4 L / c)\right] .
\end{aligned}
$$

where we have denoted with $\omega$ the optical frequency. Note that motions of $C_{1}$ and $D_{1}$ are already canceled in this subtraction, because the two MZs sense their motions equally, due to the fact that $\left|A D_{1}\right|=\left|B D_{1}\right|=\left|A C_{1}\right|=$ $\left|B C_{1}\right|$. Similarly, we have a combination of the other two MZs:

$$
\begin{aligned}
\delta \phi_{A_{2}}-\delta \phi_{B_{2}}= & \sqrt{2} \omega / c\left[x_{B}(t)-x_{A}(t)-x_{B}(t-4 L / c)\right. \\
& \left.+x_{A}(t-4 L / c)\right] .
\end{aligned}
$$

As a consequence, the total combination

$$
\phi_{\mathrm{DFI}} \equiv\left[\delta \phi_{A_{1}}-\delta \phi_{B_{1}}\right]-\left[\delta \phi_{A_{2}}-\delta \phi_{B_{2}}\right] .
$$

is free from any displacement noise. This is also anticipated, because it is obvious that $A_{1}$ and $A_{2}$ sense the beam splitters in the same way, and so do $B_{1}$ and $B_{2}$.

We now calculate the response of $\phi_{\mathrm{DFI}}$ to GWs. For a particular case, with a plane GW coming directly along the $z$ axis [i.e., $\mathbf{e}_{Z}=\mathbf{e}_{z}$, cf. Eq. (2)] and

$$
\mathbf{h}^{\mathrm{TT}}(t, \mathbf{x})=h(t-z / c)\left[\mathbf{e}_{\xi} \otimes \mathbf{e}_{\xi}-\mathbf{e}_{\eta} \otimes \mathbf{e}_{\eta}\right],
$$

it is easy to argue based on octahedron's symmetry, and the way we combine the signals, that beams in all four branches, i.e., those involving $C_{1}, D_{1}, C_{2}$, and $D_{2}$, will give equal GW contributions. For the branch involving $D_{1}$, we have, according to Eq. (5):

$$
\begin{aligned}
\frac{\phi_{\mathrm{DFI}}^{\mathrm{GW}}}{\omega L / c}= & \frac{1}{2} \int_{0}^{1} d \zeta\{h[t+(2 L \zeta-\sqrt{2} L(1-\zeta)) / c] \\
& +h[t+(2 L(1+\zeta)+\sqrt{2} L \zeta) / c] \\
& -h[t+(2 L \zeta+\sqrt{2} L(1-\zeta)) / c] \\
& -h[t+(2 L(1+\zeta)-\sqrt{2} L \zeta) / c]\} .
\end{aligned}
$$

In the frequency domain, we have

$$
\tilde{\phi}_{\mathrm{DFI}}^{\mathrm{GW}}=i \omega \tilde{h} e^{-i \sqrt{2} \Omega L / c} /(4 \Omega)\left[(2-\sqrt{2})\left[1-e^{(4+2 \sqrt{2}) i \Omega L / c}\right]+(2+\sqrt{2})\left[e^{4 i \Omega L / c}-e^{2 \sqrt{2} i \Omega L / c}\right]\right],
$$

where $\tilde{\phi}_{\mathrm{DFI}}^{\mathrm{GW}}$ and $\tilde{h}$ are Fourier transforms of $\phi_{\mathrm{DFI}}^{\mathrm{GW}}$ and $h$. This already shows a nonvanishing response. For GWs with generic propagation directions and polarizations, we use the following notation [cf. Eq. (2) and Fig. 2]:

$$
\begin{gathered}
\mathbf{e}_{X}=\mathbf{e}_{x} \cos \theta \cos \varphi+\mathbf{e}_{y} \cos \theta \sin \varphi-\mathbf{e}_{z} \sin \theta, \\
\mathbf{e}_{Y}=-\mathbf{e}_{x} \sin \varphi+\mathbf{e}_{y} \cos \varphi, \\
\mathbf{e}_{Z}=\mathbf{e}_{x} \cos \varphi \sin \theta+\mathbf{e}_{y} \sin \theta \sin \varphi+\mathbf{e}_{z} \cos \theta .
\end{gathered}
$$

In low frequencies, the GW response of the DFI combination is $\sim f^{2}$, with [4]

$$
\begin{aligned}
{\left[\tilde{\phi}_{\mathrm{DFI}}^{\mathrm{GW}}\right]_{\frac{\Omega L}{c} \ll 1}=} & 4 \sqrt{2} / 3(\Omega L / c)^{2}(\omega L / c) \\
& \times\left[\tilde{h}_{\times}\left(1+\cos ^{2} \theta\right) \cos 2 \varphi+2 \tilde{h}_{+} \cos \theta \sin 2 \varphi\right] .
\end{aligned}
$$

For general frequencies and generic incoming GW, the analytical formula for the transfer function is very complicated. Instead, as in Ref. [2], we show the root-mean- square response function, averaged over $\mathrm{GW}$ propagation direction and polarization angle, in Fig. 3.

$2 D$ Configuration. - For experimental tests, it is desirable to have a 2D configuration, which can be obtained by "squashing" our 3D configuration (Fig. 1), as shown in Fig. 4. It also consists of four MZs, $A_{1}, A_{2}$ (inner MZs, solid lines in the figure), $B_{1}$, and $B_{2}$ (outer MZs, dashed lines in the figure). Similar to the 3D configuration, the subtraction of $B_{1}$ from $A_{1}$ cancels displacements of $C_{1}$ and $D_{1}$; subtraction of $B_{2}$ from $A_{2}$ cancels displacements of $C_{2}$ and $D_{2}$. The combination of all four MZs, with appropriate time delays, will cancel motions of the beam splitters. However, as further calculations indicate, the low-frequency GW sensitivity of this 2D configuration is $\sim f^{3}$ [3].

Concluding Remarks. - This Letter brings displacement-noise-free interferometry from conceptual plausibility $[1,2]$ to concrete and practical optical designs. We provided simple interferometer configurations that realize DFI without composite mirrors. Compared with the conceptual design in Ref. [2], these are far more straightforward to implement. Moreover, our 3D configuration has superior low-frequency sensitivity $\left(\sim f^{2}\right.$, which cannot be 


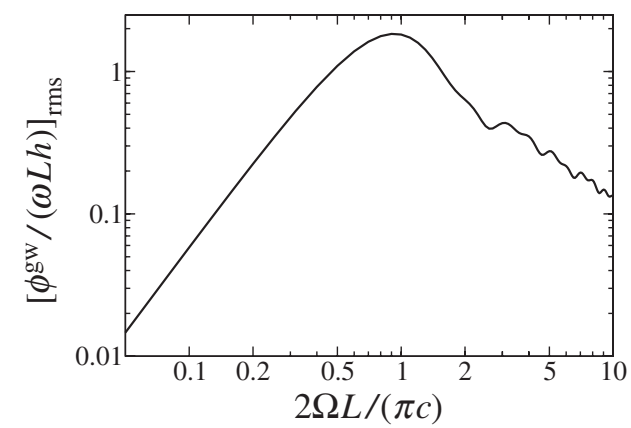

FIG. 3. Root-mean-square transfer function of the 3D, fourMZ configuration.

exceeded by any DFI configurations describable within the framework of Ref. [2]) compared to 2D configurations (which cannot exceed $\sim f^{3}$ ).

Without displacement noise, ideal DFI configurations are shot-noise-limited at all frequencies - sensitivity can be improved by simply turning up optical power. For $f \gtrsim$ $f_{c} \equiv c / L$, our 3D configuration have comparable shotnoise-limited sensitivity to conventional configurations with similar arm lengths and optical power, but without optical cavities; for $f \lesssim f_{c}$, shot-noise-limited sensitivity degrades as $\left(f / f_{c}\right)^{2}$. However, taking into account current techniques of displacement-noise suppression, we have not found a situation where DFI can improve existing detector sensitivities. In particular, for space-based detectors (for which $f_{c}$ lies in the detection band), DFI is useful if the frequency band in which test-mass acceleration noise dominates over shot noise extends from dc to frequencies above $f_{c}$. This does not apply to LISA [5], in which acceleration noise dominates only for frequencies much lower than $f_{c}$, due to the superb drag-free system to be employed. For ground-based detectors with arm lengths limited to several kilometers, it also seems very difficult for current DFI configurations to compete with current interferometers, which use optical cavities to increase the effective arm length, and target at gravitational waves at $\lesssim \mathrm{kHz}$ frequencies (with $f / f_{c} \lesssim 10^{-2}$ ) - until significant further improvements of DFI are made. Nevertheless, since in this Letter DFI has been put into such simple forms, we speculate that it will eventually find applications in future detectors, or at least become one factor to consider during the invention of new detectors. For example, cavities can be inserted to DFI configurations to enhance sensitivity. In addition, we could also apply DFI to matter-wave interferometry [6], note that (i) the proposed atomic interferometers already have MZ configurations, and (ii) with much shorter arms, displacement noise is likely to become a challenging issue for these detectors.

In practice, cancellation of displacement noises is limited by accuracies at which our idealizing assumptions are satisfied, including (for 3D configuration): (i) all arms have equal lengths, (ii) beam splitters are 50/50, (iii) mirrors are

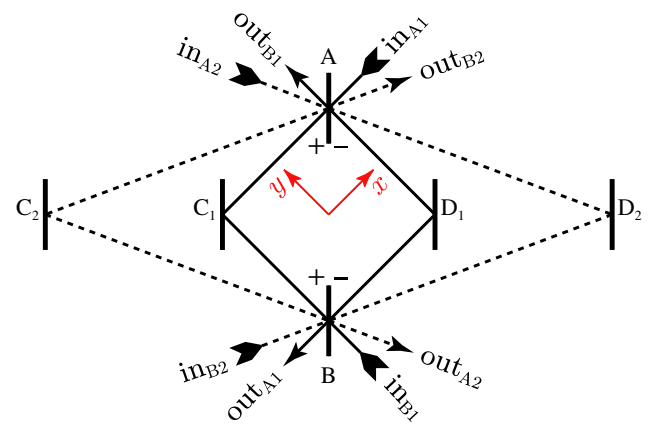

FIG. 4 (color online). The two-dimensional four-MZ configuration.

lossless, and (iv) beams incident on each mirror must all center at the same point. Effects (i)-(iii) will limit the displacement-noise reduction factor (in amplitude) at $\max \left(\delta L / L,\left|R_{\mathrm{BS}}-T_{\mathrm{BS}}\right|, \epsilon\right)$, where $\delta L$ is length accuracy, $R_{\mathrm{BS}}$ and $T_{\mathrm{BS}}$ are the beam splitters' power reflectivity and transmissivity, and $\epsilon$ is the loss of the mirrors (in power). It is reasonable to expect a 100 to 1000 -fold reduction in displacement noise. Effect (iv) will give rise to an extra noise of $\sim \delta \theta \delta l$, where $\delta l$ is the distance between centers of the spots, and $\delta \theta$ is noise in mirror tilt. If mirror tilt and translational displacement noise are related by the mirror size $a$, then we would expect a limiting reduction factor of $\delta l / a$, which can be made comparable to or lower than the first three effects.

We thank Curt Cutler for brining Octahedron to our attention. The research of Y. C., A.P. and K. S. is supported by Alexander von Humboldt Foundation's Sofja Kovalevskaja Programme (funded by the German Federal Ministry of Education and Research). R. L. W., K. G., and E.E. M. are supported by the U.S. National Science Foundation (NSF) under Cooperative Agreement No. PHY-0107417. K. G. and E. E. M. are also supported by NSF Grant No. PHY-0457264.

[1] S. Kawamura and Y. Chen, Phys. Rev. Lett. 93, 211103 (2004).

[2] Y. Chen and S. Kawamura, Phys. Rev. Lett. 96, 231102 (2006).

[3] Y. Chen et al. (to be published).

[4] This angular response, although it looks similar to that of a Michelson interferometer lying on the $x-y$ plane, in fact cannot be put into that form, regardless of the orientation of the interferometer. In particular, the response of a Michelson interferometer with arms along $x$ and $y$ is $\propto\left[2 \tilde{h}_{\times} \cos \theta \sin 2 \varphi-\tilde{h}_{+}\left(1+\cos ^{2} \theta\right) \cos 2 \varphi\right]$; that of Michelson interferometer rotated by $45^{\circ}$ from this one is $\left[\tilde{h}_{\times}\left(1+\cos ^{2} \theta\right) \sin 2 \varphi+2 \tilde{h}_{+} \cos \theta \cos 2 \varphi\right]$.

[5] K. Danzmann and A. Rüdiger, Classical Quantum Gravity 20, S1 (2003).

[6] R. Y. Chiao and A. D. Speliotopoulos, J. Mod. Opt. 51, 861 (2004). 